\title{
Improving Educational Outcomes in Fetal Alcohol Spectrum Disorder Through Interagency Collaboration
}

\author{
Christopher J. Boys ${ }^{1}$ • Judith Bjorke ${ }^{2}$ Kathryn N. Dole ${ }^{2}$ - Cathy Dalnes ${ }^{2}$. \\ Sue Terwey $^{3} \cdot$ Pi-Nian Chang ${ }^{1}$
}

Received: 23 June 2015 /Revised: 10 December 2015 / Accepted: 11 January 2016 / Published online: 4 February 2016

(C) American Academy of Pediatric Neuropsychology 2016

\begin{abstract}
Children with fetal alcohol spectrum disorders (FASD) demonstrate a significant array of neurocognitive impairments including deficits in intelligence, attention, processing speed, executive functions, language functions, visualspatial abilities, memory, and academic achievement. Neuropsychological impairments in children and adolescents with prenatal exposure to alcohol follow a continuum from no identifiable effects at one end to pervasive impairments across areas at the other end (i.e., Spohr, Willms and Steinhausen 1993; Streissguth, Randels and Smith 1991). Due to the continuum of impairments, interagency collaboration was developed to address the special educational needs of the children. The current paper has several purposes: (1) to allow school district staff who were offered an opportunity to share their perspectives and expertise on FASD and special need issues facing their students diagnosed with FASD; (2) to identify the school and social challenges faced by students diagnosed with FASD; (3) to describe the collaborative interagency program and potential impact on classroom ecology; and (4) to determine if the interagency collaboration had an effect on intervention practices of educators within the classroom.
\end{abstract}

Christopher J. Boys

boys0009@umn.edu

1 Department of Pediatrics, University of Minnesota, Minneapolis, MN, USA

2 Minneapolis Public Schools, Minneapolis, MN, USA

3 Minnesota Organization for Fetal Alcohol Syndrome, St. Paul, MN, USA
Keywords Fetal alcohol spectrum disorder · Interagency collaboration $\cdot$ Educational outcomes $\cdot$ Classroom eology

\section{Introduction}

It is well known that alcohol is a teratogen that has an impact on a child's cognition, physical development, and behavioral functioning. Children with fetal alcohol spectrum disorders (FASD) demonstrate a significant array of neurocognitive impairments including deficits in intelligence, attention, processing speed, executive functions, language functions, visual-spatial abilities, memory, and academic achievement (Mattson et al. 2010).

Fetal alcohol spectrum disorder is an overarching term used to classify individuals who have a range of physical abnormalities and neuropsychological deficits related to prenatal alcohol exposure (Davis, Desrocher and Moore 2011; Mattson et al. 2010). The spectrum includes fetal alcohol syndrome (FAS), partial fetal alcohol syndrome (partial FAS), and alcohol-related neurodevelopmental disorder (ARND). FASD diagnoses are based on several features: (1) prenatal alcohol exposure, (2) growth deficits, (3) characteristic facial features, and (4) central nervous system (CNS) dysfunction (structural or functional) (Astley 2004; Chudley et al. 2005; Hoyme et al. 2005; National Center on Birth Defects and Developmental Disabilities, Center for Disease Control and Department of Health and Human Services [CDC], 2004).

Fetal alcohol exposure is the single most prevalent cause of intellectual impairment in children (Astley et al. 2000; Spohr et al. 1993). A most recent prevalence study found the rate of overall FASD to be approximately $3.6 \%$ of the study population (May et al. 2014). Children with FASD often have special learning needs and a wide range of behavioral challenges that can have an enormous impact on educational outcomes. Neuropsychological impairments in children and adolescents 
with prenatal exposure to alcohol follow a continuum from no identifiable effects at one end to pervasive impairments across areas at the other end (i.e., Spohr et al. 1993; Streissguth Aase et al. 1991). As a result of this demanding continuum, Minneapolis Public Schools, the University of Minnesota Fetal Alcohol Spectrum Disorders Program, and the Minnesota Organization on Fetal Alcohol Syndrome (MOFAS) developed an interagency collaboration to address the special educational needs of the children.

The current paper has several purposes: (1) to allow school district staff who were offered an opportunity to share their perspectives and expertise on FASD and special need issues facing their students diagnosed with FASD; (2) to identify the school and social challenges faced by students diagnosed with FASD; (3) to describe the collaborative interagency program and potential impact on classroom ecology; and (4) to determine if the interagency collaboration had an effect on intervention practices of educators within the classroom.

\section{Background}

Prevalence rates of the full range of FASD beyond FAS, including ARND and other alcohol-related birth defects, are believed to occur about three times as often as FAS. The Centers for Disease Control and Prevention (CDC) has estimated that FAS occurs at a rate of 0.2 to 1.5 per 1000 children. A most recent prevalence study found the rate of overall FASD to be approximately $3.6 \%$ of the study population (May et al. 2014). These approach the latest estimated prevalence of autism spectrum disorders.

New regulations at the Federal level, under both the Keeping Children and Families Safe Act of 2003, which amended and reauthorized the Child Abuse Prevention and Treatment Act (CAPTA), as well as the Individuals with Disabilities Education Improvement Act of 2004, which requires early intervention referral for at-risk children, are increasing awareness of the needs of children diagnosed with FASD. These regulations certainly result in increased numbers of children in early intervention systems who have been diagnosed with FASD and require more tailored early intervention.

Although the focus of FASD research has predominantly been on primary disabilities (i.e., attention concerns, memory problems, learning disabilities, neurocognitive sequelea), several previous studies have addressed secondary disabilities. Although research has established that there are neurocognitive deficits associated with prenatal alcohol exposure, research has been less focused on the continuum of effects and possible interventions to prevent the ongoing impairments often seen in children with FASD. Children diagnosed with FASD commonly exhibit behavior problems, social skills deficits, emotional difficulties, and poor executive functioning (e.g., organization, inhibitory control, monitoring). The significant level of secondary disabilities that severely impair the quality of life in these individuals is extremely costly to society (Connor and Streissguth 1996).

Streissguth et al. (1991) utilized the construct of secondary disabilities to encompass measurable difficulties that individuals with FASD face as they mature into young adults. Some of the common secondary disabilities that are associated with FASD include disrupted school experiences, problems with alcohol and other drug abuse, joblessness, homelessness, mental health problems, victimization, difficulties with the law, and premature death (Streissguth 1997). Many of the secondary disabilities that these individuals experience are exacerbated by weaker community service delivery and the lack of management of an individualized education or treatment plan.

Despite its prevalence, FASD is often referred to as a "hidden" disability, owing to the common absence of overt physical characteristics (e.g., facial dysmorphology) in affected individuals. It is also not uncommon for children diagnosed with an FASD to demonstrate weak adaptive skills (including social, emotional, and self-regulation skills), as well as reduced cognitive functioning due to their disorder. However, regardless of a child's outward presentation, it is believed that irreversible brain damage underlies a diagnosed child's inability to achieve academic and developmental milestones on par with typically developing peers. Schools may be overwhelmed by overt special needs while more "invisible" disorders (such as "mild" organic brain damage associated with FASD) may be missed or mistaken as emotional or behavioral issues. This outlook has the potential to offset even the best-intentioned intervention services for children diagnosed with FASD as they are likely to be mislabeled based solely on academic assessments and may be misidentified on the autism spectrum or overlooked altogether due to the invisible nature of the comorbid symptoms of the disorder. It is essential for schools to consider the overall neurocognitive profile and develop appropriate educational intervention services in order to maximize academic outcomes.

Teachers and other education-related practitioners are charged with the critical task of supporting children's development and academic success regardless of individual levels of function. With the Interagency Collaboration Model, the Diagnostic Clinic provides the conceptualization of neuropsychological strengths and weaknesses, while education staff bring their skill sets and specific strategies aimed at improved engagement and better academic outcomes for students. In addition to the teachers' skills of adapting or modifying their classrooms and curriculum as well as their teaching styles to address each student's needs, their experiences in working with special needs children are integral to creating and implementing successful intervention strategies for children diagnosed with FASD. According to the Substance Abuse and Mental Health Services Administration (SAMHSA) of the Department of Health and Human Services, 80 to 100 students out of 10,000 have learning problems related to 
FASD. The majority of these students are enrolled in general education classrooms (FASD Center for Excellence 2007). It is hoped that due to the complexity of needs of students diagnosed with FASD in school districts, educators will contribute to the needs assessment with their first-hand knowledge of working with children diagnosed with FASD. Alternately, for clinical practitioners who often report little to no experience working in an educational setting, insights they may provide based on their experience with the underlying neuropsychological profile will be informative to the educator's abilities to adequately address special needs in the classroom.

A thorough comprehensive evaluation for children with an FASD can assist in uncovering the hidden nature of the disability and associated invisible special needs, which could be missed or misinterpreted by even experienced educators. Once the educators have access to the students' neuropsychological profile, they can more effectively create an educational plan incorporating this information.

\section{Stakeholders}

The Minneapolis Public Schools (MPS), along with the University of Minnesota FASD (UMNFASD) Program and the MOFAS, began an interagency collaboration to address many of the issues surrounding the needs of children with FASD in the educational setting. Often, interagency collaborations arise out of a recognized community, medical, or educational need for a certain population or from working relationships among colleagues who recognize the opportunity for collaboration even though they work in different environments. This was the case for the current project. The community collaboration between MOFAS and the UMNFASD program initially was focused on addressing the needs of adjudicated youth in the court system and recognizing that schools play an important role in the success of these young people. At the same time, a MPS staff member was working both in special education and with internationally adopted children at the University of Minnesota. Minnesota has long been recognized as having one of the highest rates of international adoptions per capita, and with increased numbers of these children presenting with prenatal exposure, the need for educational programs to address the effects of prenatal alcohol exposure was increasing. These two factors led to a recognized need for more focus on educational outcomes for children diagnosed with FASD.

The Interagency Collaborative FASD Project identified over 200 students with a documented diagnosis of FASD. Table 1 shows "snapshots" of active students by disability status identified through special education assessment each spring over a 4-year period. Although numbers may vary considerably each year, MPS has consistently seen at least $10 \%$ of its students diagnosed with FASD not eligible or not assessed for special education services.

\section{Model}

There were several reasons that the Interagency Collaborative FASD Project began.There was a recognized need for improved educational services for students with FASD and for clinical evaluators to better understand the educational environment and programming in the schools. Increasing public awareness through advocacy by MOFAS led to an observed increase in parent requests for services for MPS students with FASD. Several goals were developed following the formation of the Interagency Collaborative FASD Project. First and foremost, the goal was to improve educational outcomes for students with FASD. In addition, developing a partnership to support families
Table 1 Special education primary disability category

\begin{tabular}{|c|c|c|c|c|}
\hline & Year 1 & Year 2 & Year 3 & Year 4 \\
\hline No special education & $6(10 \%)$ & $15(19 \%)$ & $16(14 \%)$ & $14(15 \%)$ \\
\hline Other Health Disability (OHD) & $28(44 \%)$ & $27(33 \%)$ & $34(30 \%)$ & $41(43 \%)$ \\
\hline Emotional/behavioral disorder (EBD) & $9(14 \%)$ & $13(16 \%)$ & $29(25 \%)$ & $14(15 \%)$ \\
\hline Developmental delay (DD/ECSE) & $8(13 \%)$ & $10(12 \%)$ & $14(12 \%)$ & $9(9 \%)$ \\
\hline SNAP (MPS problem-solving model) ${ }^{\mathrm{a}}$ & $7(11 \%)$ & $9(11 \%)$ & $11(10 \%)$ & $10(11 \%)$ \\
\hline Specific learning disability (SLD) & $3(5 \%)$ & $4(5 \%)$ & $8(7 \%)$ & $4(4 \%)$ \\
\hline Developmental cognitive disability (DCD) & $2(3 \%)$ & $1(1 \%)$ & $2(2 \%)$ & $2(2 \%)$ \\
\hline Speech/language impaired & 0 & $1(1 \%)$ & $1(1 \%)$ & 0 \\
\hline Total students & 63 & 81 & 115 & 95 \\
\hline
\end{tabular}

${ }^{a}$ Students needing alternative programming (SNAP). Minneapolis Public Schools use alternative evaluation procedures to identify some students' eligibility for special education that meet federal standards. These students would otherwise qualify under the Minnesota disability categories of specific learning disability (SLD) and developmental cognitive disability (DCD) - mild to moderate range 
seeking resources for their children, as well as providing support for educators, was needed. Another important goal was to expand the knowledge of MPS staff about individual needs of students with FASD and with the characteristics of the overall profile associated with FASD. Additional goals of the project were to assist in determining appropriate eligibility for special education services and to develop educational and behavioral strategies to support students with FASD.

To address the complexity of managing the interagency workings and to provide direct support to staff and families of students in our schools, a liaison social worker position was developed. This position was endorsed by the UMNFASD Program, but the initial budget was provided to MPS by MOFAS. After the second year, MPS took over the responsibility for the funding.

\section{Methods}

A qualitative approach was used to gather information relating to FASD in the school system through semi-structured anonymous online survey interviews with educators and other school practitioners. In the initial survey, district staff were offered an opportunity to share their perspectives and expertise on FASD and special needs issues facing their students. Annual surveys were completed by individuals who were involved with staff development trainings and other services provided by the MPS, UMNFASD, or MOFAS partners. Analysis of participant reports focused on extracting thematic information which would highlight or improve professionals' understanding of the following: (1) capacities of schools to address FASDs through understanding their approach to special needs in general; (2) Who are the key FASD stakeholders in schools?; and (3) the usefulness of an FASD diagnosis for educators.

\section{Results}

The Interagency Collaborative FASD Project identified over 200 students with a documented diagnosis of FASD. Table 1 shows snapshots of active students by disability status identified through special education assessment each spring over a 4 -year period. Although numbers may vary considerably each year, MPS has consistently seen at least $10 \%$ of its students diagnosed with FASD not eligible or not assessed for special education services.

Table 2 shows the federal setting (FS) of students as indicated by their level of service on the Individualized Education Plan (IEP). FS 1 means that students are taught in a regular education classroom, with less than $21 \%$ of their time in a special education classroom or setting. FS 2 indicates that students are seen in a resource or self-contained setting for $21-60 \%$ of their school day. Students receiving services at
Table 2 Federal setting (FS) of students identified

\begin{tabular}{lllll}
\hline & Year 1 & Year 2 & Year 3 & Year 4 \\
\hline No special ed & $6(10 \%)$ & $15(19 \%)$ & $16(14 \%)$ & $14(15 \%)$ \\
FS 1 & $19(30 \%)$ & $15(19 \%)$ & $25(22 \%)$ & $21(22 \%)$ \\
FS 2 & $7(11 \%)$ & $10(12 \%)$ & $15(13 \%)$ & $18(19 \%)$ \\
FS 3 & $18(29 \%)$ & $20(25 \%)$ & $23(20 \%)$ & $16(17 \%)$ \\
FS 4 & $3(5 \%)$ & $8(10 \%)$ & $22(19 \%)$ & $11(12 \%)$ \\
Early childhood & $5(8 \%)$ & $8(10 \%)$ & $8(10 \%)$ & $5(5 \%)$ \\
Transition plus & $5(8 \%)$ & $4(5 \%)$ & $3(3 \%)$ & $10(11 \%)$ \\
Homebound & - & $1(1 \%)$ & - & - \\
\hline
\end{tabular}

the level of FS 3 are served in a separate classroom (all students here receive special education) for more than $60 \%$ of their day. The most restrictive setting, FS 4 , is a separate public school facility that only serves students receiving special education for more than $50 \%$ of their day. The numbers below show that many of our identified students are located in the general education classroom for most of the day (includes no special education services and FS 1 and 2).

District teachers and staff were asked to indicate which of these were the biggest challenges faced by students with diagnosed or suspected FASD. In the initial survey conducted at the beginning of the project, the special education staff indicated the prevalence of both school challenges and social challenges that they observed in children diagnosed with FASD. In year 1, 32 surveys were completed.

\section{School Challenges}

- $80 \%$ Impulsivity, distractibility

- $72 \%$ Slow processing speed

- $57 \%$ Memory concerns

- $55 \%$ Lack of cause-effect reasoning

- $46-51 \%$ Academics (writing, math, reading)

- $43 \%$ Language

- $30 \%$ Sensory

- $26 \%$ Fine motor

\section{Social Challenges}

- $52 \%$ Chemical health in family

- $52 \%$ Mental health in family

- $46 \%$ Multiple moves

- $38 \%$ School moves

- $43 \%$ safety concerns

- $41 \%$ In foster care

- $37 \%$ With relative

- $28 \%$ In adoptive home

- $22 \%$ Homeless 
Each spring, staff who participated in some way with the project were asked to complete a survey. Table 3 shows where survey respondents were located. The highest percentage of surveys was returned from the K-5 programming, followed by grades 6-8.

Using the surveys completed in the spring of year 1 as a baseline, there were notable gains in strategies attempted in the classroom in succeeding years, as seen in Table 4. The greatest increase (30 percentage points) was in teachers reporting that they were modifying the classroom environment to meet the needs of their students with FASD. This was followed by providing planned breaks and the use of social stories (21 point increases), using a five-point scale to assist with self-regulation (18 point gain), and 15 point gains in the areas of "thinking younger" (working with the student at a developmentally younger level), allowing more processing time and providing sensory opportunities. The modification of the environment will most allow students to begin to utilize their strengths and minimize their weaknesses. Planned breaks give students an opportunity to rest from a taxing task and to re-group or start over if needed. Social stories assist in preparing for stressful or novel events, while the five-point scale helps students identify when their behavior or emotions are both within a normal range or escalating to a level requiring intervention. Allowing the students to have more processing time often is key to reducing frustration for the students, which in turn reduces the number of outbursts in the classroom.

\section{Additional Project Efforts}

Additionally, several other changes occurred as a result of the interagency collaboration. This included district-wide and school-based training opportunities that were conducted primarily by the UMNFASD program. These trainings focused on training of the educational professionals regarding characteristics of FASD and appropriate interventions. In addition, case managers of identified students were contacted each fall

Table 3 Annual survey respondents, year 2-4

\begin{tabular}{llll}
\hline & $\begin{array}{l}\text { Year 2 } \\
(n=111)\end{array}$ & $\begin{array}{l}\text { Year 3 } \\
(n=58)\end{array}$ & $\begin{array}{l}\text { Year 4 } \\
(n=31)\end{array}$ \\
\hline Early childhood & $20 \%$ & $16 \%$ & $26 \%$ \\
Grades K-5 & $35 \%$ & $24 \%$ & $61 \%$ \\
Grades 6-8 & $27 \%$ & $19 \%$ & $19 \%$ \\
Grades 9-12 & $9 \%$ & $14 \%$ & $7 \%$ \\
FS 1 and 2 & $18 \%$ & $10 \%$ & $19 \%$ \\
EBD (FS 3 and 4) & $23 \%$ & $22 \%$ & $8 \%$ \\
SNAP/SLD/DCD (FS3) & $21 \%$ & $21 \%$ & $16 \%$ \\
Autism (FS3) & $8 \%$ & $2 \%$ & - \\
Transition plus (18-21 years old) & $4 \%$ & $4 \%$ & $3 \%$ \\
\hline
\end{tabular}

by the MPS FASD Liaison. They were provided information about the Interagency Collaboration Project, a resource list of references relevant to understanding the disability, and learning and behavioral strategies that may be more effective in meeting the needs of their students. Clinicians from the UMNFASD program along with the MPS FASD Liaison participated in IEP and other school meetings to discuss the results of the neuropsychological evaluation and to assist in intervention planning and development of recommendations for the IEP. Finally, project staff worked with MPS Early Childhood and Transition Plus staff to discuss program development, including more comprehensive screening to identify children with prenatal alcohol exposure and distribution of educational and community resource materials.

Another aspect of the collaboration was to incorporate a neuropsychological evaluation for students in Minneapolis Schools with an FASD diagnosis. While the purpose of this paper is on the impact of the collaboration on classroom ecology, for individual students the evaluation was critical to assist in IEP development and intervention planning at the individual student level. The full neuropsychological assessment provided a conceptualization for strengths and weaknesses and allowed for a more accurate interpretation of the presenting behaviors.

\section{Discussion}

The survey format yielded some very important discussions and afforded opportunities to focus on participant expertise when it was most relevant. As expected, the participants of the program provided a wealth of knowledge pertaining to the status of FASD in schools. Educators are adept at identifying and providing effective interventions for students with special needs. However, there are complexities identified within the students with FASD in which it is very important to have the correct conceptualization based on the neuropsychological assessment. Many educators that completed the survey indicated they had some knowledge of FASD. As the project progressed, it was the combination of additional trainings provided by UMNFASD clinicians and MOFAS staff, matching interventions to the neuropsychological profile, and targeted IEP meetings in which the educators could deploy the correct combination of already existing classroom interventions to better match the needs of the complex students with FASD.

Despite a high percentage of educators indicating some previous knowledge of FASD, the majority of participants responded positively to the idea of being further educated on FASDs. The desire for increased professional development opportunities for educators was a common theme. In addition, despite reporting a high level of previous knowledge, the participants still made changes to their approaches following work with the Interagency Collaborative Project. 
Table 4 Percentage of respondents of spring surveys reporting strategies attempted in classrooms

\begin{tabular}{lllll}
\hline & Year 1 $(n=32)$ & Year 2(n=111) & Year 3(n=58) & Year 4(n=31) \\
\hline Modify environment & 41 & 56 & 67 & 71 \\
Think younger & 59 & 63 & 64 & 74 \\
Structure and consistency & 66 & 77 & 76 & 77 \\
Routines & 59 & - & - & - \\
Short and simple & 69 & 75 & 76 & 77 \\
Abstract made concrete & 63 & 68 & 74 & 71 \\
Breaks & 53 & 63 & 72 & 74 \\
More processing time & 66 & 77 & 76 & 81 \\
Sensory opportunities & 56 & 65 & 62 & 71 \\
Demonstrate/model & - & 68 & 74 & 74 \\
Frequent repetition & - & 72 & 74 & 71 \\
Visual cues & - & 69 & 63 & 68 \\
5-Point scale & - & 30 & 31 & 48 \\
Social stories & - & - & 34 & 55 \\
Average & 59 & 65 & 65 & 70 \\
\hline
\end{tabular}

Overall, improving educational outcomes for students with special needs in the classroom is a key area of concern for all involved. A majority of educators interviewed in the current project expressed an increase in awareness, as well as making effective changes to classroom ecology to address both student and teacher needs. An interesting aspect of this issue is highlighted by the high number of participants who, as a result of involvement in the project, had utilized different approaches to intervene with their students diagnosed with FASD.

There are several remaining areas that stakeholders have addressed that were impacted by the Interagency Collaboration.

1. FASD Awareness and Education in Schools

Awareness of the prevalence of children with FASD in the schools promotes discussion and knowledge sharing among professionals by expanding education initiatives. There are few school district-wide initiatives for training and support on FASD in Minnesota. Although there are several FASD workshops as well as group and community initiatives in Minneapolis and greater Minnesota offering support services, there has been very little focus on translating the clinical knowledge and FASD research into educational practice. Rather than focusing on individual schools as the children present to FASD Diagnostic Clinics, a broader approach for training by interagency collaborations will provide a more sustainable intervention model. Community agencies with expertise should provide FASD consultation support to interested schools. Additionally, consideration should be given to training appropriate school staff on screening for prenatal alcohol exposure while conducting social histories and educational profiles when student concerns have been noted. This might also include those students who, on the surface, present with disruptive behaviors.
2. FASD as a Comorbid Disorder

Given the heterogeneity of the neurocognitive profile of FASD, it is important to recognize FASD as an independent, but comorbid disorder most often associated with learning and/or behavioral disorders. It is important that a designation of Other Health Disability (OHD) be utilized to maximize extra support for children in schools. Children with FASD are often seen solely as impulsive and disruptive, and the underlying etiology of the behaviors is not addressed. When FASD is seen as a medical diagnosis, similar to a type of acquired brain injury, there can be less frustration expressed by adults and more creative interventions implemented.

3. FASD-Specific Interventions

Whereas the neuropsychological assessment of FASD is well established, interventions specific to children with FASD are not as established. It may not be, however, that many new specific interventions for FASD may be required. Rather, the vast array of educational strategies already developed can be deployed; they need to be correctly matched to the neuropsychological profile. Children with FASD are often misunderstood as having conduct disorder, behavior problems, or learning difficulties. The involvement of the UMNFASD program with the schools provided education professionals with neuropsychological profiles for the students. In addition, educators were able to educate UMNFASD clinicians as to how interventions may look in the classroom setting. This collaboration allowed for even greater specificity and effectiveness of the classroom interventions. Further collaboration that can be developed is between the clinicians and school psychologists. School psychologists already have vast knowledge in assessment and the development of classroom interventions. Good communication 
between the clinical neuropsychologists and school psychologists can quickly translate into the development of targeted classroom interventions than can be deployed across the district.

4. Advocacy for Children with FASD

The project identified that many neuropsychological evaluations that were completed by the UMNFASD program were not previously made available to educators for a variety of reasons. It is important that an FASD Diagnostic Clinic should work closely with and continue to advocate for effective partnerships and information sharing between referral sources, caregivers of diagnosed children, and educational teams. Case consultations and support for caregivers, teachers, and schools of children diagnosed with FASD can be facilitated by a community partner such as MOFAS. Educators are in a unique role of improving clinicians' understandings of due process requirements and aspects of classroom programming from an educator's point of view. This aspect also can assist in improving communication between schools and families and eliminate some of the conflict that can occur if parents and educators are frustrated.

5. Longevity of an Interagency Collaboration

Interagency collaborations are critical for effective interventions in profiles as complex as that of FASD. Success requires organization and continuity across multiple areas due to having the public school, a diagnostic clinic, and a community-based program. An absolutely critical component was the establishment of a social worker/liaison position. In the current project, MOFAS initially provided the funding, but once the positive nature of the position was established, the MPS eventually assumed the cost of the position after seeing the benefits. In addition, over the course of 3 years, the social worker assumed a greater role from within the school setting and began to work independently at IEP meetings and responded to requests from schools for training and education regarding FASD. This allowed for the UMNFASD clinicians to again focus on assessments, as well as district-wide training, and take a lesser role in the daily workings of the student programming.

6. Include a Full Neuropsychological Assessment as Part of FASD Assessments

A comprehensive assessment is critical to providing a profile of strengths and weaknesses of the students diagnosed with FASD. This profile of strengths and weaknesses can help to illustrate how learning disabilities and behavior problems are interrelated with FASD. With a more comprehensive neurocognitive profile, the educational professionals were more effectively able to tailor interventions to address the unique needs of many students with FASD and conceptualize the comorbid symptoms that may have previously been misunderstood.

\section{Conclusions}

There is little doubt that, in order to adequately address the needs of students with FASD, there has to be a unified team of professionals available to provide care and supports for children diagnosed with FASD and their families. This timeline includes diagnosis to intervention to follow-up. While the Interagency Collaborative Project was able to contribute to the development of more comprehensive and inclusive supports for children with FASD in schools, it was also the aim to work within and streamline the established framework currently set up in schools and local communities. Key community institutions such as Public School systems, FASD clinics, and community partners such as MOFAS must consider how to develop effective communication mechanisms to ensure that multi-disciplinary consulting and supports are in place to adequately support students diagnosed with FASD. Finally, one of the key components of the current program was the liaison social worker position that coordinated the activities of all of the collaborators and was available to work from within the education system.

While there are benefits to the collaboration, there are also limitations within the current study. For example, within each year of the implementation, the pool of eligible participants changed from year to year due to specific school involvement. This created a large variability in the numbers of responders to the survey from year to year. Another limitation was the lack of use of converging data. A large district such as the one involved in the current project collects vast amounts of data regarding student performance. It will be important in future studies to use aspects of converging data, such as student gains in statewide testing, fewer disciplinary referrals, or changes to an IEP. This would be helpful in further monitoring of the program's impact.

\section{Summary}

The education practitioners working with the target population of children with FASD are the individuals on the front lines of intervention services. While the education professionals indicated a greater need for more information regarding FASD in the classroom, it was their overall understanding and vast experience in addressing the special needs of students with disabilities that allowed them to make effective changes to classroom ecology. The educators were most able to create an effective classroom ecology through the dissemination of information and FASD-specific trainings, as well as more effective communication between the schools and community partners.

FASD requires a paradigm shift in that the neuropsychological profile of FASD can be masked by behaviors. For example, attention problems are not simply attention problems in children with FASD. Rather, the attention difficulties are 
often driven by executive dysfunction that keeps the children from maintaining sequences, organizing multi-step tasks, and shifting effectively between tasks. While on the surface the issues present as attention, when interventions focus on these aspects of executive functions, the attentional difficulties are more likely to resolve. Subsequently, teachers indicated that when they were aware of the FASD profile, they were more likely to implement classroom strategies that focused on the underlying aspects rather than the presenting behavior difficulties. The neuropsychological evaluation can result in a streamlining of existing interventions that address the important aspects of the profile of FASD and reduce frustrations of the teachers and students. It is important that current research and best practices developed by children with FASD continue to be implemented within the schools. It is often difficult to move current research into practice. In the case of FASD, a collaborative project with stakeholders that includes public schools, an FASD Diagnostic Clinic, and a community advocacy partner is essential and critical for effective educational intervention of FASD.

\section{Compliance with Ethical Standards}

Human and Animal Rights and Informed Consent No animal or human studies were carried out by the authors for this article.

Conflict of Interest Christopher J. Boys, Judith Bjorke, Kathryn N. Dole, Cathy Dalnes, Sue Terwey, and Pi-Nian Chang declare that they have no conflict of interest.

\section{References}

Astley, S. J. (2004). Diagnostic guide for fetal alcohol spectrum disorders: the 4-digit diagnostic code (3rd ed.). Seattle, WA: University of Washington Publication Services.

Astley, S. J., Bailey, D., Talbot, C., \& Clarren, S. K. (2000). Fetal alcohol syndrome (FAS) primary prevention through FAS diagnosis: I. Identification of high-risk birth mothers through the diagnosis of their children. Alcohol and Alcoholism, 35(5), 499-508.
Chudley, A. E., Conry, J., Cook, J. L., Loock, C., Rosales, T., \& LeBlanc, N. (2005). Fetal alcohol spectrum disorder: Canadian guidelines for diagnosis. Canadian Medical Association Journal, 172, s1-s21.

Connor, P. D., \& Streissguth, A. P. (1996). Effects of prenatal exposure to alcohol across the life span. Alcohol Health and Research World, 20(3), 170-174.

Davis, K., Desrocher, M., \& Moore, T. (2011). Fetal alcohol spectrum disorder: a review of neurodevelopmental findings and interventions. Journal of Developmental and Physical Disabilities, 23, $143-167$.

FASD Center for Excellence (2007). Fetal alcohol spectrum disorders by the numbers. Rockville, MD: SAMHSA.

National Center on Birth Defects and Developmental Disabilities, Center for Disease Control, \& Department of Health and Human Services. (2004). Fetal alcohol syndrome: Guidelines for referral and diagnosis. Retrieved from http://www.cdc.gov/ncbddd/fas/documents/ FAS guidelines accessible.pdf.

Hoyme, H. E., May, P. A., Kalberg, W. O., Kodituwakku, P., Gossage, J. P., Trujillo, P. M., \& Robinson, L. K. (2005). A practical clinical approach to diagnosis of fetal alcohol spectrum disorders: clarification of the 1996 Institute of Medicine criteria. Pediatrics, 115, 3947.

Mattson, S.N., Roesch, S.C., Fagerlund, Å., Autti-Räm, I., Jones, K., May, P.A., \& ... Riley, E.P. (2010). Toward a Neurobehavioral Profile of Fetal Alcohol Spectrum Disorders. Alcoholism: Clinical \& Experimental Research, 34(9)

May, P., Baete, A., Russo, J., Elliott, A., Blankenship, J., Kalberg, W., Buckley, D., Brooks, M., Hasken, J., Abdul-Rahman, O., Adam, M., Robinson, L., Manning, M., \& Hoyme, E. (2014). Prevalence and characteristics of fetal alcohol spectrum disorders. Pediatrics, 134(5), 855-866.

Spohr H. L., Willms J., \& Steinhausen H. C. (1993). Prenatal alcohol exposure and long-term developmental consequences. Lancet, 10, 907-10.

Steinhausen, H., Willms, J., \& Spohr, H. (1993). Long-term psychopathological and cognitive outcome of children with fetal alcohol syndrome. Journal of the American Academy of Child \& Adolescent Psychiatry, 32(5), 990-994.

Streissguth, A. P., Randels, S. P., \& Smith, D. F. (1991). A test-retest study of intelligence in patients with fetal alcohol syndrome: implications for care. Journal of the American Academy of Child \& Adolescent Psychiatry, 30(4), 584-587.

Streissguth, A., Barr, H., Kogan, J., \& Bookstein, F. (1997). Primary and secondary disabilities in fetal alcohol syndrome. In A. Streissguth \& J. Kanter (Eds.), The challenge of fetal alcohol syndrome: overcoming secondary disabilities (pp. 25-39). Seattle: University of Washington Press. xxvii, 250 pp. 